\title{
A time-resolved X-ray powder diffraction method to trace the decomposition of $\mathrm{PdB}_{y}$ solid solutions
}

\author{
T. G. Berger ${ }^{1}$, A. Leineweber ${ }^{1, *}$, E. J. Mittemeijer ${ }^{1}$, \\ M. Knapp ${ }^{2}$
}

${ }^{1}$ Max Planck Institute for Metals Research, Heisenbergstr. 3, D-70569 Stuttgart, Germany.

${ }^{2}$ Institute for Materials Science, Darmstadt University of Technology, Petersenstr. 23, D-64287 Darmstadt, Germany.

*Contact author; e-mail: a.leineweber@mf.mpg.de

Keywords: palladium-boron alloys, miscibility gap, compositional changes, line broadening

\begin{abstract}
A powder diffraction method is presented for reconstructing probability density

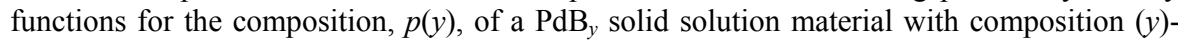
dependent lattice parameter from the simultaneous analysis of all reflections in the powder diffraction patterns. The method is applied to the decomposition of a solid solution in a miscibility gap: two 'boundary phases' appear which gradually approach but don't attain fully the compositions of the expected equilibrium phases whereas a significant amount of material with intermediate compositions remains.
\end{abstract}

\section{Introduction}

X-ray powder diffraction provides information about the atomic structure (size and symmetry of unit cell, atom positions and displacements) of crystalline materials via the positions (Bragg angles) and intensities of reflections. Analysis of the width and shape of diffraction-line profiles, furthermore, allows extraction of microstructural information, e.g. in terms of microstrain and crystallite size [1]. Analysis of this physical broadening, denoted $f$, is, however, considerably hindered by the instrumental contribution $g$, with which $f$ is convoluted leading to the real measured profile $h$. Various convolution and deconvolution methods exist to determine the physical from the measured profile [2]. The present work aims at developing a method to reconstruct complicated probability density functions for composition, $p(y)$, of a solid solution material, with composition (y)-dependent lattice parameters, from the simultaneous analysis of all reflections in the powder pattern.

The solid solution considered here is the palladium-rich solid solution $\operatorname{PdB}_{y}$. It exhibits an extraordinarily high interstitial solid solubility up to about $\mathrm{PdB}_{\sim 0.25}$ above temperatures of $440^{\circ} \mathrm{C}$ ( $y$ in $\mathrm{PdB}_{y}$ denotes both the atomic ratio and the occupancy by boron atoms of the octahedral sites in the $c c p$ palladium host lattice) as well as several low-temperature phases and two-phase fields below temperatures of about $400-450^{\circ} \mathrm{C}$ [3]. A remarkable and for binary interstitial solid solutions rare feature is the miscibility gap with a critical temperature 
of $T_{\text {crit }}=410^{\circ} \mathrm{C}$ which covers at the monotectic temperature of $T_{\text {mono }}=312^{\circ} \mathrm{C}$ a composition range of about $0.03<y<0.10$ (figure 1) [4,5]. Decomposition in this miscibility gap upon annealing can be considered as a redistribution of boron atoms over the octahedral interstices leading to changes in the probability density function for composition $p(y)$ as averaged over the sample, which shows up in a characteristic way in powder diffraction patterns.

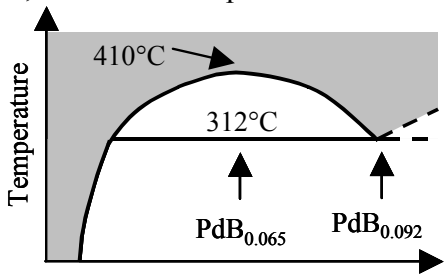

Boron content

Figure 1. Part of the Pd-B phase diagram with the miscibility gap [3]. The grey shaded area shows the phase field of the solid solution.

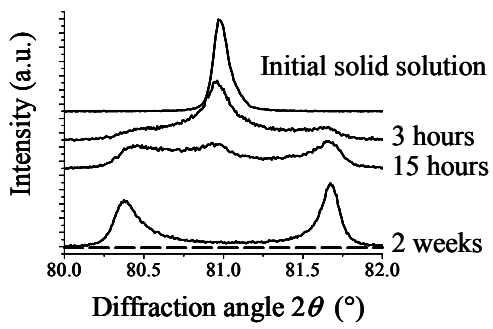

Figure 2. 311-Diffraction peak group (CuK $\alpha_{1}$ radiation) recorded from a $P d B_{0.064}$ sample annealed for various times at $340^{\circ} \mathrm{C}$.

\section{Experimental}

\section{Preparation and heat treatment}

$\mathrm{PdB}_{0.064}$ was prepared by arc melting under argon from weighted amounts of palladium foils and an appropriate amount of boron pieces. $\mathrm{PdB}_{0.050}$, previously used in a neutron diffraction study, was prepared similarly from ${ }^{11} \mathrm{~B}$ [7]. Both reguli were checked for homogeneity [5-7]. The powder samples (filed from the reguli) were sealed under vacuum in quartz capsules, annealed at $800^{\circ} \mathrm{C}$ for $5 \mathrm{~min}$ and subsequently quenched in water. The annealing was performed to relieve deformation stresses due to the filing procedure. The composition was calculated [6] by

$$
a=a_{0}+\alpha \cdot y=3.8920+0.6882 y[\AA]
$$

The heat treatments for the decomposition were performed from 3 hours till up to 8 weeks (in sealed quartz tubes encapsulated in a salt bath for up to 4 days, longer annealings in a furnace) at $340^{\circ} \mathrm{C}$ or $355^{\circ} \mathrm{C}$ with subsequent water quenching.

\section{X-ray diffraction measurements}

X-ray powder diffraction measurements of the initial, stress-relieved solid solution and of the subsequently annealed samples were performed

- in house on a Philips X'Pert MPD instrument $\left(\mathrm{CuK} \alpha_{1}\right.$ radiation in Bragg-Brentano geometry, powder samples were deposited as a thin layer together with internal silicon standard powder on $\mathrm{Si}$ wafers cut parallel to the (510) plane) and

- for two selected $\mathrm{PdB}_{0.064}$ specimen, one quenched from $800^{\circ} \mathrm{C}$ to preserve the solid solution and one annealed for 18 days at $340^{\circ} \mathrm{C}$ to evoke the decomposed state, employing synchrotron radiation with $\lambda=1.1315 \AA$ on the B2 beamline [8], HASYLAB, Hamburg (no internal standard, separate $\mathrm{LaB}_{6}$ standard (SRM 660a) measurements; powder deposited on Kapton foil, measurements performed in transmission geometry).

All reflections were recorded by measuring over $2 \theta$ (diffraction-angle) subranges sufficiently large to determine the background at both sides of each reflection (group). 


\section{Data evaluation method}

The decomposition of the initially homogeneous solid solution into a boron-rich and a boronpoor solid solution phase is clearly revealed by the occurring splitting of the Bragg reflections. For short annealing times (e.g. figure 2), the original solid solution is still present. Even after the longest applied heat-treatment times $\left(8\right.$ weeks at $340^{\circ} \mathrm{C}$ and 2 weeks at $355^{\circ} \mathrm{C}$ ), still considerable diffraction-line broadening remains which is asymmetric for both the high and the low boron components ('boundary phases') such that residual intensity between the pairs of reflections remains. To analyse the time-dependent decomposition as observed in the X-ray diffraction patterns, the reflection profiles were modelled using the program TOPAS [9] as described below.

\section{$X$-ray data evaluation}

For each heat treatment, all individually measured reflections, from $\mathrm{PdB}_{y}$ as well as from the internal standard (in house data), were merged into one data set after manually subtracting the background. This complete pattern was modelled in a Rietveld-like fashion, considering a convolution of three line-broadening contributions:

$$
h=g \otimes f_{1} \otimes f_{2}
$$

with $g$ and $f_{1}$ taken as Voigt functions with angle-dependent Gaussian and Lorentzian full width at half maximum values $B_{L}$ and $B_{G}$, respectively. The instrumental profile $g$ was determined from the internal $\mathrm{Si}$ standard (in house data) or the separately measured $\mathrm{LaB}_{6}$ standard (synchrotron data), respectively, by fitting $L Y, L X, G U, G V, G W$ according to:

$$
\text { g: } \quad B_{g, L}=L Y \tan \theta+L X / \cos \theta ; \quad B_{g, G}^{2}=G U \tan ^{2} \theta+G V \tan \theta+G W \text {. }
$$

The structural line broadening $f_{1}$ of the two quenched solid solution samples was described semi-empirically by size and anisotropic microstrain broadening, fitting $L_{1}, L_{2}, S_{1}, S_{2}, G_{2}$ :

$f_{1}: \quad B_{f_{1}, L}=L_{1} \tan \theta+L_{2} / \cos \theta$;

$$
B_{f_{1}, G}^{2}=\left(S_{1}+S_{2} \frac{h^{2} k^{2}+k^{2} l^{2}+h^{2} l^{2}}{\left(h^{2}+k^{2}+l^{2}\right)^{2}}\right) \tan ^{2} \theta+\left(G_{2} / \cos \theta\right)^{2} .
$$

The present description of the microstrain broadening anisotropy was derived from the assumption of an isotropic microstress distribution leading to anisotropic microstrain if the crystals are elastically anisotropic [10] (as is the case for Pd).

The additional line broadening occurring in the diffraction patterns of the annealed specimen, $f_{2}$, can be attributed to the occurrence of decomposition-induced compositional variations described by $p(y)$, starting from a delta-shaped distribution for the initial solid solution. For the annealed samples $p(y)$ was approximated by a frequency polygon (figure 3) with composition steps of $\Delta y=0.001$. Due to the composition dependence of the lattice parameters of the cubic crystal lattice (equation (1)) of $\mathrm{PdB}_{y}, p(y)$ reflects itself directly in each reflection [11]. In Topas [9] this was incorporated in terms of a multiphase refinement of 120 'phases' with composition values $y^{\prime}$ which are spaced by $\Delta y$. Each 'phase' $\mathrm{PdB}_{y^{\prime}}$ ' has been ascribed a compositional line broadening contribution corresponding to a trapezoid (shaded in grey in figure 3) with a width of $2 \alpha \Delta y \tan \theta / a$ (cf. equation (1)), and an area (integral intensity) proportional to $\left(p\left(y^{\prime}\right)+p\left(y^{\prime}+\Delta y\right)\right) / 2$. 


\section{Penalty functions to achieve stability in refinements of $p(y)$}

Refining the $p\left(y^{\prime}\right)$ values without any constraints leads to severe correlations between different $p\left(y^{\prime}\right)[12,13]$. These correlations can be reduced to achieve physically realistic 'smooth' $p(y)$ by application of so-called penalty functions $(P F)$. These serve as contribution to the overall $\chi_{\text {pen }}{ }^{2}$ (penalised least squares), additional to the usual $\chi^{2}$ from the difference between observed and the calculated profile [14]:

$$
\chi_{p e n}^{2}=\chi^{2}+(K \cdot P F)^{2} \quad K: \quad \text { penalty weighting factor [9] }
$$

Commonly $[12,13]$, these penalty functions are modified integrals of the first or second derivatives of $p(y)$, e.g. as used here:

$$
\begin{array}{lll}
P F_{1}=\int q_{1} \cdot\left(p^{\prime}(y)\right)^{2} d y & q_{1}: & p^{-1}(y) . \\
P F_{2}=\int q_{2} \cdot\left(p^{\prime \prime}(y)\right)^{2} d y & q_{2}: & \left(C^{2}+\left(p^{\prime \prime}(y)\right)^{2}\right)^{-1} .
\end{array}
$$

A compromise for the weight $K$ of the penalty function contribution to the overall $\chi^{2}$ must be found to obtain a reasonable $p(y)$ : In case of too low weight the correlations are not sufficiently suppressed; in case of a too high weight, physical features in the shape of $p(y)$ may be suppressed ('oversmoothing') [13]. In the present case, the application of the penalty function was allowed to increase the $\chi^{2}$ value by not more than $10 \%$ as compared to the value obtained with an unconstrained refinement. For most of the performed refinements, $P F_{1}$ produced satisfactory results; $P F_{2}$ (with another constant $C$ to be adjusted empirically) was only used for the refinement of the microstrain broadening change (see Results).

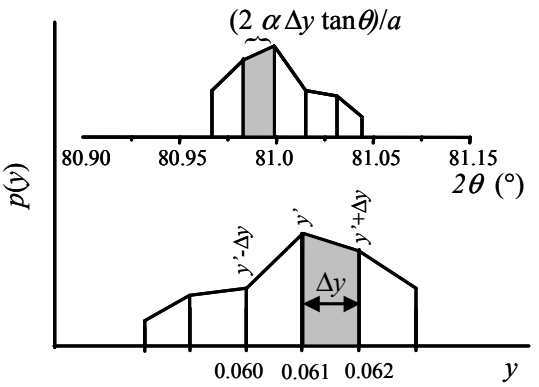

Figure 3. Frequency polygon type description of the probability density function of composition $p(y)$ and the corresponding line-broadening $f_{2}$ for the 311-reflection. The grey area shows the contribution associated with composition $y=0.061$ (with an area proportional to $\left.\left(p\left(y^{\prime}\right)+p\left(y^{\prime}+\Delta y\right) / 2\right)\right)$.

\section{Results and Discussion}

Results for $p(y)$ obtained with the described method are shown in figures $4 \mathrm{a}-\mathrm{c}$ for $\mathrm{PdB}_{0.064}$ and $\mathrm{PdB}_{0.050}$ annealed for various times at $340^{\circ} \mathrm{C}$ and $\mathrm{PdB}_{0.050}$ annealed for various times at $355^{\circ} \mathrm{C}$.

Initially, $p(y)$ contains a remaining contribution from the original solid solution. Its phase fraction was determined by subtracting an estimated parabolic 'background $p(y)$ ' between the two 'boundary phases' (figure 4a). The time-dependence of the molar phase fraction (referring to the formula $\mathrm{PdB}_{y}$ ) of the parent solid solution is similar for the three samples, but the $\mathrm{PdB}_{0.064}$ sample decomposes much faster than the $\mathrm{PdB}_{0.050}$ at the same temperature of $340^{\circ} \mathrm{C}$. Further, $\mathrm{PdB}_{0.050}$ decomposes faster at $340^{\circ} \mathrm{C}$ than at $355^{\circ} \mathrm{C}$ (figure 5). Therefore the driving force for the decomposition has its maximum in the middle of the miscibility gap. 
The values of the boundary (product) phase compositions (figure 6) were determined by a parabolic fit to the three locally highest $p\left(y^{\prime}\right)$ values in the range of the local maxima pertaining to the high and low boron product phases. For short annealing times the $p(y)$ of the $\mathrm{PdB}_{0.050}$ sample, annealed at $355^{\circ} \mathrm{C}$, did not show clear local maxima for the 'boundary phases', therefore the two shortest annealing times were omitted. For long annealing times, differences in the 'boundary phase' composition for the same temperature occur, which are not expected by the phase diagram (cf. figure 6).
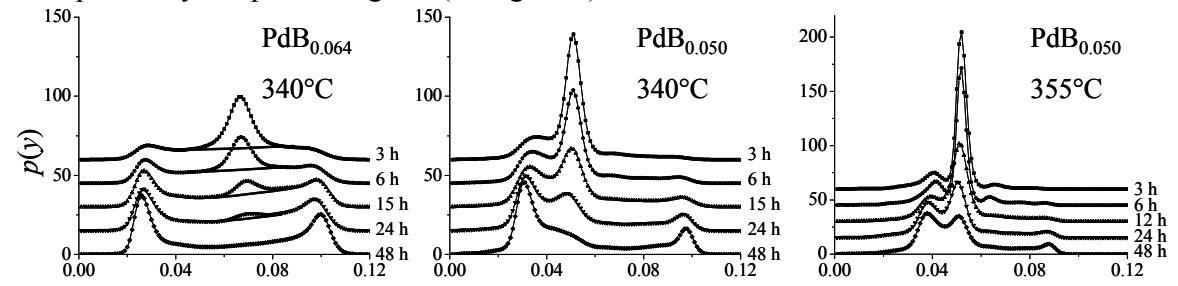

Boron content according to $\mathrm{PdB}_{y}$

Figure 4a-c. p(y) for different annealing times and different initial compositions. Clearly visible is the faster decomposition for $P d B_{0.064}$ compared to $P d B_{0.050}$ and for $340^{\circ} \mathrm{C}$ compared to $355^{\circ} \mathrm{C}$ (estimation of the fraction of initial solid solution has been indicated in $4 a$ ).

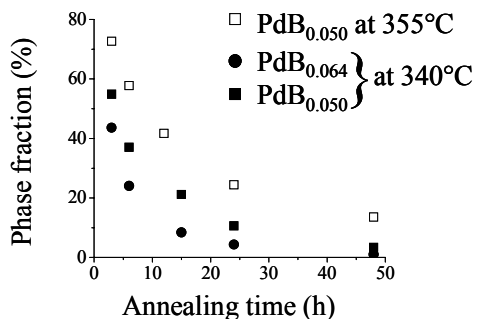

Figure 5. Relative phase fractions of the initial solid solutions vs. annealing time.

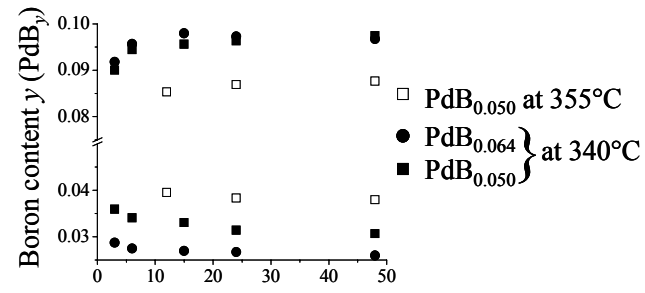

Annealing time (h)

Figure 6. Boundary product phase compositions vs. annealing time. For $P d B_{0.050}$ at $355^{\circ} \mathrm{C}$ the shorter annealing times could not be analysed (see text).

So far in the analysis the line profile parameters of the microstructural line-broadening, $f_{1}$, were kept constant and taken equal to those determined for the initial solid solution. Since the mechanical microstrain broadening is anisotropic (cf. equation (4)), it should be possible to detect a change in $f_{1}$ upon decomposition, given sufficient resolution of the data, as it was the case for the synchrotron measurements. Unconstrained refinement of $S_{1}$ and $S_{2}$ (equation (4)) was not possible due to the correlation of the $S_{1}$ parameter with the also $\tan \theta$-dependent line broadening due to $p(y)$. Therefore the ratio $S_{1} / S_{2}$ was fixed to the value of the initial solid solution. By stepwise increasing $S_{1}$ and $S_{2}$ it could be shown that there is indeed an increase of microstrain broadening upon decomposition. The first derivative as used in $P F_{1}$ has its highest values at the inflection points of $p(y)$ located at the 'flanks' of $p(y)$, thus the penalty function may suppress subtle width-related effects. Therefore, for the analysis of $S_{1}$ and $S_{2}$, the penalty function $P F_{2}$ was employed which has its highest values at the maxima of $p(y)$. The resulting $p(y)$ are shown in figure 7 for the initial solid solution values of $S_{1}$ and $S_{2}$ characterising the anisotropic microstrain broadening and for $5 S_{1}$ and $5 S_{2}$; in the last case a distinctly improved fit to the measured diffraction pattern was achieved. It follows that 
changes in $S_{1}$ and $S_{2}$ influence $p(y)$ : broader $f_{1}$ due to higher values of $S_{1}$ and $S_{2}$ leads to a 'sharpened' $p(y)$ (figure 7).

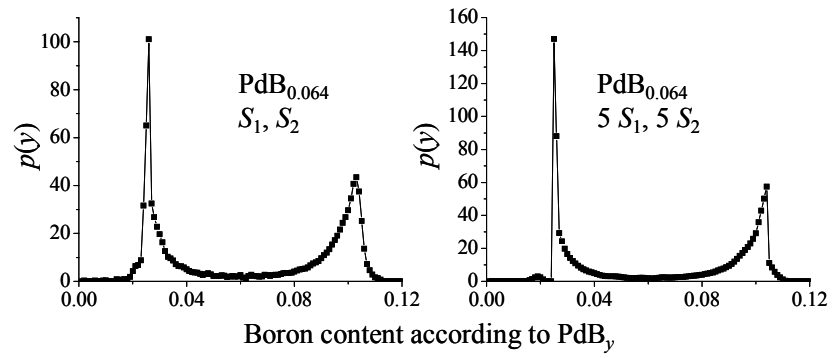

\section{Conclusions}

Figure 7. $p(y)$ as determined from synchrotron data adopting microstrain broadening parameters $S_{1}$ and $S_{2}$ equal to those of the solid solution (left) and 5 times as large (right) $\left(\mathrm{PF}_{2}\right.$ applied).

A method has been presented to determine presuppositionlessly composition distributions of decomposing solid solutions from complicated diffraction-line profile shapes in an simultaneous evaluation of all reflections in a powder diffraction pattern. This composition distribution is determined by convolution fitting of a probability density function $p(y)$ with an instrumental broadening contribution $g$ measured by internal standards and a microstrain and size broadening contribution $f_{1}$ measured from a parent solid solution sample.

\section{References}

1. Delhez, R., de Keijser, Th. H., Mittemeijer, E. J., 1982, Fresenius Z. Anal. Chem. 312 (1), 1.

2. Snyder, R. L. \& Fiala, J. \& Bunge, H.-J, 1999, Defect and Microstructure Analysis by Diffraction (Oxford University Press), chapter 26 -27.

3. Liao, P. K., Spear, K. E., Schlesinger, M. E., 1996, J. Phase Equilib. 17, 340.

4. Alqasmi, R. A., Brodowsky, H., Schaller, H.-J., 1982, Z. Metallkde. 73, 331.

5. Beck, M., Mittemeijer, E. J., 2001, Z. Metallkde. 92, 1271.

6. Beck, M., Ellner, M., Mittemeijer, E. J., 2001, Acta Mater. 49, 985.

7. Berger, T. G., Leineweber, A., Mittemeijer, E. J., Fischer, P., 2004, Phys. Stat Sol. A 201, 1484.

8. Knapp, M., Baehtz, C., Ehrenberg, H., Fuess, H., 2004, J. Synchrotron Rad. 11, 328.

9. TOPAS, General Profile and Structure Analysis Software for Powder Diffraction Data, V2.0, Bruker AXS GmbH, Karlsruhe, Germany.

10. Stokes, A. R., Wilson, A. J. C., 1944, Proc. Phys. Soc. 56, 174.

11. Leineweber, A., Mittemeijer, E. J., 2004, J. Appl. Cryst. 37, 123.

12. Good, I. J., Gaskins, R. A., 1971, Biometrica 58, 255.

13. Louboutin, R., Louër, D., 1969, Acta Cryst. A 25, 335.

14. Coelho, A. A., 2000, J. Appl. Cryst. 33, 899. 